\title{
On the Apparent Non-Uniqueness of the Electromagnetic Field Components of Return Strokes Revisited
}

\author{
Vernon Cooray ${ }^{1}$, Gerald Cooray ${ }^{2}$, Marcos Rubinstein ${ }^{3}$ (I) and Farhad Rachidi ${ }^{4, *(D)}$ \\ 1 Department of Electrical Engineering, Uppsala University, 75237 Uppsala, Sweden; \\ vernon.cooray@angstrom.uu.se \\ 2 Great Ormond Street Hospital (GOSH), Great Ormond Street, London WC1N 3JH, UK; gerald.cooray@ki.se \\ 3 Institute for Information and Communication Technologies, University of Applied Sciences and Arts Western \\ Switzerland, 1401 Yverdon-les-Bains, Switzerland; marcos.rubinstein@heig-vd.ch \\ 4 Electromagnetic Compatibility Laboratory, Swiss Federal Institute of Technology (EPFL), \\ 1015 Lausanne, Switzerland \\ * Correspondence: farhad.rachidi@epfl.ch
}

Citation: Cooray, V.; Cooray, G.; Rubinstein, M.; Rachidi, F. On the Apparent Non-Uniqueness of the Electromagnetic Field Components of Return Strokes Revisited. Atmosphere 2021, 12, 1319. https://doi.org/ 10.3390/atmos12101319

Academic Editor: Sergey Pulinets

Received: 1 September 2021

Accepted: 3 October 2021

Published: 9 October 2021

Publisher's Note: MDPI stays neutral with regard to jurisdictional claims in published maps and institutional affiliations.

Copyright: (c) 2021 by the authors. Licensee MDPI, Basel, Switzerland. This article is an open access article distributed under the terms and conditions of the Creative Commons Attribution (CC BY) license (https:// creativecommons.org/licenses/by/ $4.0 /)$.

\begin{abstract}
Recent research work shows that there are four procedures that can be used to calculate the electromagnetic fields from a current source. These different procedures, even though producing the same total field, give rise to field components that differ from one procedure to another. This has led to the understanding that the various field terms that constitute the total field cannot be uniquely determined. In this paper, it is shown that all four field expressions can be reduced to a single field expression, and the various field terms arising from acceleration, uniformly moving, and stationary charges can be uniquely determined. The differences in the field terms arising from different techniques are caused by the different ways of summing up the contribution to the total electric field coming from the accelerating, moving, and stationary charges.
\end{abstract}

Keywords: dipole fields; radiation; induction; electrostatic; velocity fields; Coulomb fields; field components; lightning; return stroke

\section{Introduction}

The students of electromagnetic theory are introduced, as a standard, to electromagnetic fields generated by an electric dipole when they are taught about the electromagnetic radiation [1]. It is shown that the dipole electric fields can be separated into electrostatic, induction, and radiation terms, even though one cannot assign any clear physical significance to these field terms except for the radiation. An interesting question that one can raise in this context is the following: Are these field components non-unique, or is there another way to express the total fields so that the physical processes that give rise to the dipole fields become apparent? A similar problem exists in the case of calculating the electromagnetic fields of more complex sources, for example, the return strokes in lightning flashes.

In lightning research, return stroke models are utilized to estimate the electromagnetic fields at different distances generated by return strokes. These return stroke models specify the spatial and temporal variation of the charge and current associated with the return stroke. Once this information is specified, it is possible to calculate the electromagnetic fields using Maxwell's equations (e.g., [2-7]). However, once the spatial-temporal distributions of the return stroke charge and current are specified, there are several ways that the Maxwell's equations can be utilized in calculating the electromagnetic fields. At present, there are four methods developed in the literature to evaluate the electromagnetic fields once the spatial and temporal distribution of the current are given [8,9]. These are known as the dipole (Lorentz) technique, the continuity equation technique, and two versions of the procedures based on moving and accelerating charges. All these techniques give rise to the 
same total field, but the various components that constitute that total field are apparently different in each technique. On the other hand, the processes that generate different field components in an electromagnetic field are clearly specified in classical electrodynamics. For example, the electric and magnetic fields generated by any time-varying spatial distribution of currents and charges can be separated into two parts [1]: the radiation fields and the Coulomb fields. The radiation fields are generated by accelerating charges, and the Coulomb fields are generated by stationary and uniformly moving charges. In the case of uniformly moving charges, the Coulomb field has to be modified to take into account the charge movement, and these modified Coulomb fields are known as velocity fields. Given these clear specifications as to the cause of electromagnetic fields, it is interesting to investigate why the various field components associated with the total fields differ from each other in different techniques, whereas the physical processes that generate the electromagnetic fields are the same irrespective of which technique is used in extracting the electromagnetic field. For this reason, we have decided to take a closer look at this problem, and the results of this investigation are presented here. Even though the presented derivations and the associated discussion concern the specific case of lightning-generated fields, the results of the paper can be extended to any type of source.

\section{Electric Fields Evaluated Using Standard Techniques}

In this section, we will review the various expressions for the electric fields from a vertical lightning channel obtained using standard techniques. We will concentrate only on the electric fields, but the conclusions to be made in this study are also valid for the magnetic fields.

Thottappillil et al. [7] described two independent approaches to calculate the electromagnetic fields from a lightning channel. They also described two other procedures, but the resulting field expressions obtained using these two other procedures can be shown to be analytically equivalent to the two former procedures. For this reason, those field expressions are not considered in the current study.

Here, we consider a return stroke located over a perfectly conducting ground plane. The effect of the ground plane on the electromagnetic fields is taken into account using the concept of images. The geometry relevant to the field expressions to be introduced is given in Figure 1. The return stroke channel is assumed to be straight and vertical. The spatial and temporal distribution of the current flowing along the return stroke channel is specified as follows: the current flowing at a point located at a height $z$ along the channel is given by $i(z, t)$. The point $\mathrm{P}$, located over the perfectly conducting ground, is the reference point where the field expressions for the electromagnetic fields are given. The $z$-axis is directed out of the ground plane, and the electric fields directed along the $z$-axis are considered positive. The speed of propagation of the return stroke front at height $z$ is denoted by $u_{z}$ which, in general, is a function of $z$.

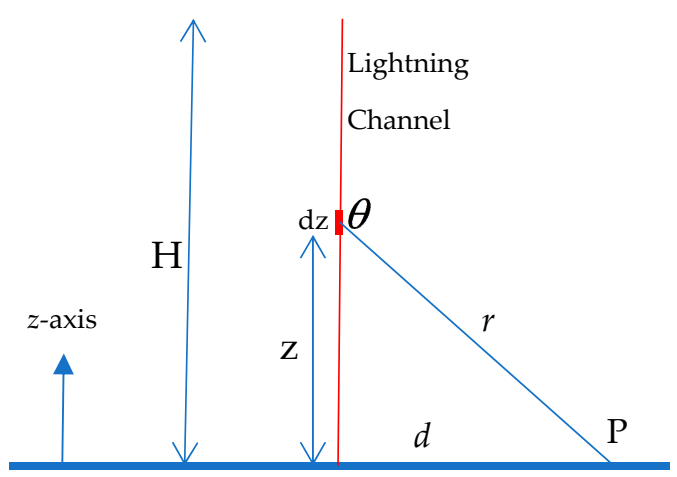

Figure 1. Geometry relevant to the calculation of electromagnetic fields from a return stroke. 


\subsection{Lorentz Condition or Dipole Procedure}

As outlined in [8], this method involves the following steps in deriving the expression for the electric field:

(i) The specification of the current density $J$ of the source.

(ii) The use of $J$ to find the vector potential $A$.

(iii) The use of $A$ and the Lorentz condition to find the scalar potential $\varphi$.

(iv) The computation of the electric field $E$ using $A$ and $\varphi$.

In this technique, the source is described only in terms of the current density, and the fields are described in terms of the current. The final expression for the electric field at point $\mathrm{P}$ based on this technique is given by

$$
\begin{aligned}
& E_{z}(t)=\frac{1}{2 \pi \varepsilon_{0}} \int_{0}^{L} \frac{2-3 \sin ^{2} \theta}{r^{3}} \int_{t_{b}}^{t} i\left(z, \tau^{\prime}\right) d \tau d z+\frac{1}{2 \pi \varepsilon_{0}} \int_{0}^{L} \frac{2-3 \sin ^{2} \theta}{c r^{2}} i\left(z, t^{\prime}\right) d z \\
& -\frac{1}{2 \pi \varepsilon_{0}} \int_{0}^{L} \frac{\sin ^{2} \theta}{c^{2} r} \frac{\partial i\left(z, t^{\prime}\right)}{\partial t} d z
\end{aligned}
$$

The three terms in (1) are the well-known static, induction, and radiation components. In the above equation, $t^{\prime}=t-r / c, \tau^{\prime}=\tau-r / c, t_{b}$ is the time at which the return stroke front reaches the height $z$ as observed from the point of observation $P, L$ is the length of the return stroke that contributes to the electric field at the point of observation at time $t, c$ is the speed of light in free space, and $\varepsilon_{0}$ is the permittivity of free space. Observe that $L$ is a variable that depends on time and on the observation point. The other parameters are defined in Figure 1.

\subsection{Continuity Equation Procedure}

This method involves the following steps as outlined in [8]:

(i) The specification of the current density $J$ (or charge density $\rho$ of the source).

(ii) The use of $J$ (or $\rho$ ) to find $\rho$ (or $J$ ) using the continuity equation.

(iii) The use of $J$ to find $A$ and $\rho$ to find $\varphi$.

(iv) The computation of the electric field $E$ using $A$ and $\varphi$.

The expression for the electric field resulting from this technique is the following.

$$
E_{z}(t)=-\frac{1}{2 \pi \varepsilon_{0}} \int_{0}^{L} \frac{z}{r^{3}} \rho\left(z, t^{\prime}\right) d z-\frac{1}{2 \pi \varepsilon_{0}} \int_{0}^{L} \frac{z}{c r^{2}} \frac{\partial \rho\left(z, t^{\prime}\right)}{\partial t} d z-\frac{1}{2 \pi \varepsilon_{0}} \int_{0}^{L} \frac{1}{c^{2} r} \frac{\partial i\left(z, t^{\prime}\right)}{\partial t} d z
$$

\section{Electric Field Expressions Obtained Using the Concept of Accelerating Charges}

Recently, Cooray and Cooray [9] introduced a new technique to evaluate the electromagnetic fields generated by time-varying charge and current distributions. The procedure is based on the field equations pertinent to moving and accelerating charges. According to this procedure, the electromagnetic fields generated by time-varying current distributions can be separated into static fields, velocity fields, and radiation fields. In that study, the method was used to evaluate the electromagnetic fields of return strokes and current pulses propagating along conductors during lightning strikes. In [10], the method was utilized to evaluate the dipole fields and the procedure was extended in [11] to study the electromagnetic radiation generated by a system of conductors oriented arbitrarily in space. In [12], the method was applied to separate the electromagnetic fields of lightning return strokes according to the physical processes that give rise to the various field terms. In a study published recently, the method was generalized to evaluate the electromagnetic fields from any time-varying current and charge distribution located arbitrarily in space [13]. These studies led to the understanding that there are two different ways to write the field expressions associated with any given time-varying current distribution. The two procedures are named as (i) the current discontinuity at the boundary procedure or discontinuously 
moving charge procedure and (ii) the current continuity at the boundary procedure or continuously moving charge procedure [13]. The field expressions resulting from these two procedures are given in the next two subsections.

\subsection{Current Discontinuity at the Boundary or Discontinuously Moving Charge Procedure}

Assume, as before, that the return stroke channel is straight and vertical. The vertical direction coincides with the $z$-axis. Consider a channel element $d z$ located at height $z$ from ground level. One can visualize the current propagation in this element as follows: The current is initiated at the bottom of the element and, after propagating along the element, it is terminated at the other end of the element. The current and the return stroke speed remain the same as it propagates along the channel element. The changes in the current or speed as a function of height are taken into account at the boundary of the adjacent elements. That is, the current that is being terminated in one element and the speed of propagation along that element are slightly different to the current and the speed that are being initiated in the adjacent element located above. In other words, the change in the current and speed is visualized to take place at the boundaries of the channel elements. By making the size of the elements infinitesimal, it is possible to take into account the continuous variation of current and speed along the channel. This procedure is depicted in Figure 2I. With this picture, one can write down the field terms resulting from the current initiation and termination. By treating the whole channel as a sum of small current elements, the total field can be obtained by integrating the field terms corresponding to the current elements along the channel. The resulting field equations were derived by Cooray and Cooray [12], and the resulting electric field separated into radiation, velocity and static terms is given by

$$
\begin{array}{r}
\mathbf{E}_{z, r a d}(t)=-\int_{0}^{L} \frac{d z}{2 \pi \varepsilon_{0} c^{2} r}\left\{\frac{\partial i\left(z, t^{\prime}\right) \sin ^{2} \theta}{\partial t}\right\}+\int_{0}^{L} \frac{d z}{2 \pi \varepsilon_{0} c^{2} r}\left\{\frac{2 u_{z} \sin ^{2} \cos \theta}{r\left(1-\frac{u}{c} \cos \theta\right)} i\left(z, t^{\prime}\right)\right\} \\
-\int_{0}^{L} \frac{d z}{2 \pi \varepsilon_{0} c^{2} r}\left\{\frac{u_{z}^{2} \sin ^{4} \theta}{r c\left(1-\frac{u_{z}}{c} \cos \theta\right)^{2}} i\left(z, t^{\prime}\right)\right\}+\int_{0}^{L} \frac{d z}{2 \pi \varepsilon_{0} c^{2} r}\left\{\frac{u_{z} \cos \theta \sin ^{2} \theta}{\left(1-\frac{u_{z}}{c} \cos \theta\right)} i\left(z, t^{\prime}\right)\right\} \\
\mathbf{E}_{z, \text { vel }}(t)=\int_{0}^{L} \frac{i\left(z, t^{\prime}\right) d z}{2 \pi \varepsilon_{0} r^{2}\left[1-\frac{u_{z}}{c} \cos \theta\right]^{2}}\left\{\frac{\cos \theta}{u_{z}}-\frac{1}{c}\right\}\left[1-\frac{u_{z}^{2}}{c^{2}}\right] \\
\mathbf{E}_{z, \text { stat }}(t)=-\int_{0}^{L} \frac{d z}{2 \pi \varepsilon_{0} r^{2}}\left\{-\frac{\cos ^{2} \theta}{c} i\left(z, t^{\prime}\right)+\frac{\cos \theta}{u_{z}} i\left(z, t^{\prime}\right)\right\}+\int_{0}^{L} \frac{d z}{2 \pi \varepsilon_{0} r^{2}}\left\{\frac{\left\{3 \sin ^{2} \theta-2\right\}}{r} \int_{0}^{t} i\left(z, \tau^{\prime}\right) d \tau\right\}
\end{array}
$$

In the field expressions, the first term (Equation (3a)) is the radiation field coming from accelerating charges, the second term (Equation ( $3 b)$ ) is the velocity field, and the third term (Equation (3c)) is the field term resulting from stationary charges.

\subsection{Current Continuity at the Boundary or Continuously Moving Charge Procedure}

Consider again the channel element $d z$. In this procedure, the current crossing the boundary of the element is continuous, and changes in the current take place inside the channel element. This procedure is depicted in Figure 2II. If the source is such that there is a current discontinuity at a boundary (i.e., at the point of initiation of a return stroke or at the end of the channel), then it has to be treated separately. If the current and the speed do not vary with height, then there is no charge accumulation or charge acceleration taking place inside this channel element. On the other hand, if the current and the speed vary within the element, then the charge accumulation and acceleration or deceleration take place inside the volume. Accordingly, this element will contribute to the static, the velocity, 
and the radiation field terms. The expression for the electric field of the return stroke based on this procedure and separated again into radiation, velocity, and static terms is given by

$$
\begin{gathered}
\mathbf{E}_{z, \text { rad }}=-\int_{0}^{L} \frac{\sin \theta d z}{2 \pi \varepsilon_{0} c^{2} r\left[1-\frac{u_{z} \cos \theta}{c}\right]}\left[u z \frac{\partial i\left(z, t^{\prime}\right)}{\partial z}-\frac{\partial i\left(z, t^{\prime}\right)}{\partial t}+i\left(z, t^{\prime}\right) \frac{\partial u_{z}}{\partial z}\right]-\frac{i\left(0, t^{\prime}\right) u_{z}(0)}{2 \pi \varepsilon_{0} c^{2} d} \\
\mathbf{E}_{z, v e l}=\int_{0}^{L} \frac{d z i\left(0, t^{\prime}\right)\left\{1-\frac{u_{z}^{2}}{c^{2}}\right\}}{2 \pi \varepsilon_{0} r^{2}\left[1-\frac{u_{z}}{c} \cos \theta_{z}\right]^{2}}\left[\frac{\cos \theta}{u_{z}}-\frac{1}{c}\right] \\
\mathbf{E}_{z, \text { stat }}=\int_{0}^{L} \frac{d z \cos \theta \int_{0}^{t}\left[\frac{\partial i\left(0, t^{\prime}\right)}{\partial z}-\frac{1}{u_{z}} \frac{\partial i\left(0, t^{\prime}\right)}{\partial t}\right] d \tau}{2 \pi \varepsilon_{0} r^{2}}
\end{gathered}
$$

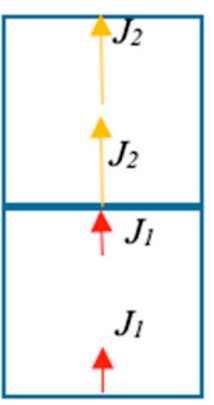

I

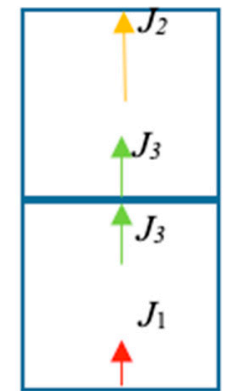

II

Figure 2. The difference between the two procedures to evaluate the electromagnetic fields using the field expressions for accelerating and moving charges. Each subfigure shows two adjacent channel elements. In procedure (I), called the current discontinuity at the boundary procedure or the discontinuously moving charge procedure, the changes of current and velocity take place at the boundary of the two elements, while they remain constant within each volume. In this procedure, charges are accumulated at the boundary of the two elements if the current changes in space. In procedure (II), which is called the current continuity at the boundary procedure or the continuously moving charge procedure, the current and velocity change as they pass through the element but remain continuous at the boundary. Thus, no charges are accumulated at the boundary. Adapted from [13].

Note that in this procedure, the current across the boundary of each element is continuous, with the possible exceptions, as mentioned earlier, of the lower boundary of the channel element at the ground and the upper boundary of the last channel element. This discontinuity in the current has to be taken into account separately in the derivation, and it will give rise to an additional radiation term. The last term in Equation (4a) is the radiation field resulting from any discontinuity at ground level (this term is also referred to as the turn-on term [14]. A discontinuity at the top of the return stroke channel would result in a similar expression). In this expression, $u_{z}(0)$ is the return stroke speed at ground level and $d$ is the horizontal distance from the strike point to the point of observation.

Observe that even though the field terms were separated purely based on the physical processes that gives rise to them, the radiation, velocity, and static terms given above appear different to the corresponding field expressions obtained using the discontinuously moving charge procedure. 


\section{Electromagnetic Field Expressions Corresponding to the Transmission Line Model of Return Strokes}

In the analysis to follow, we will discuss the similarities and differences of the different techniques described in the previous section by adopting a simple model for lightning return stroke, namely the transmission line model [15]. The equations pertaining to the different considered techniques presented in Section 3 will be particularized for the transmission line model.

In the transmission line model, the return stroke current travels upwards with constant speed and without attenuation. This model selection will not compromise the generality of the results to be obtained because, as we will show later, any given spatial and temporal current distribution can be described as a sum of current pulses moving with constant speed without attenuation and whose origins are distributed in space and time. Let us now particularize the general field expressions given earlier to the case of the transmission line model. In the transmission line model, the spatial and temporal distribution of the return stroke is given by

$$
\begin{aligned}
& i(z, t)=0 \quad t<z / v \\
& i(z, t)=i(0, t-z / v) \quad t \geq z / v
\end{aligned}
$$

In the above equation, $i(0, t)$ (for brevity, we write this as $i(t)$ in the rest of the paper) is the current at the channel base and $v$ is the constant speed of propagation of the current pulse. One can simplify the field expressions obtained in the continuity equation method and in the continuously moving charge method by substituting the above expression for the current in the field equations. The resulting field equations are given below. However, observe, as we will show later, that the field expressions corresponding to the Lorentz condition method or the discontinuously moving charge method remain the same under the transmission line model approximation.

\subsection{Dipole Procedure (Lorentz Condition)}

The expression for the electric field obtained using the dipole procedure in the case of the transmission line model is given by Equation (1) except that $i(z, t)$ should be replaced by $i(t-z / v)$. The resulting equation with $t^{\prime}=t-z / v-r / c$ is:

$$
E_{z}(t)=\frac{1}{2 \pi \varepsilon_{0}} \int_{0}^{L} \frac{2-3 \sin ^{2} \theta}{r^{3}} \int_{t_{b}}^{t} i\left(\tau^{\prime}\right) d \tau d z+\frac{1}{2 \pi \varepsilon_{0}} \int_{0}^{L} \frac{2-3 \sin ^{2} \theta}{c R^{2}} i\left(t^{\prime}\right) d z-\frac{1}{2 \pi \varepsilon_{0}} \int_{0}^{L} \frac{\sin ^{2} \theta}{c^{2} R} \frac{\partial i\left(t^{\prime}\right)}{\partial t} d z
$$

\subsection{Continuity Equation Procedure}

In the case of the transmission line model $[8,16] \rho\left(z, t^{\prime}\right)=i\left(0, t^{\prime}-z / v\right) / v$. Substituting this in the field expression (2) and using straightforward trigonometric manipulations, we obtain

$$
\begin{gathered}
E_{z}(t)=-\frac{1}{2 \pi \varepsilon_{0}} \int_{0}^{L} \frac{\cos \theta}{r^{2}} \frac{i\left(t^{\prime}-z / v\right)}{v} d z-\frac{1}{2 \pi \varepsilon_{0}} \int_{0}^{L} \frac{\cos \theta}{c r v} \frac{\partial i\left(t^{\prime}-z / v\right)}{\partial t} d z \\
-\frac{1}{2 \pi \varepsilon_{0}} \int_{0}^{L} \frac{1}{c^{2} r} \frac{\partial i\left(t^{\prime}-z / v\right)}{\partial t} d z
\end{gathered}
$$

Note that all the field terms are now given in terms of the channel-base current.

\subsection{Discontinuously Moving Charge Procedure}

In the case of the transmission line model, the field equations pertinent to this procedure can be written as follows.

$$
\begin{gathered}
\mathbf{E}_{z, r a d}(t)=-\int_{0}^{L} \frac{d z}{2 \pi \varepsilon_{0} c^{2} r}\left\{\frac{\partial i\left(t^{\prime}\right) \sin ^{2} \theta}{\partial t}\right\}+\int_{0}^{L} \frac{d z}{2 \pi \varepsilon_{0} c^{2} r}\left\{\frac{2 v \sin ^{2} \theta \cos \theta}{r\left(1-\frac{v}{c} \cos \theta\right)} i\left(t^{\prime}\right)\right\} \\
-\int_{0}^{L} \frac{d z}{2 \pi \varepsilon_{0} c^{2} r}\left\{\frac{v^{2} \sin ^{4} \theta}{r c\left(1-\frac{v}{c} \cos \theta\right)^{2}} i\left(t^{\prime}\right)\right\}+\int_{0}^{L} \frac{d z}{2 \pi \varepsilon_{0} c^{2} r}\left\{\frac{v \cos \theta \sin ^{2} \theta}{\left(1-\frac{v}{c} \cos \theta\right)} i\left(t^{\prime}\right)\right\}
\end{gathered}
$$




$$
\begin{gathered}
\mathbf{E}_{z, \text { vel }}(t)=-\int_{0}^{L} \frac{i\left(t^{\prime}\right) d z}{2 \pi \varepsilon_{0} r^{2}\left[1-\frac{v}{c} \cos \theta\right]^{2}}\left\{\frac{\cos \theta}{v}-\frac{1}{c}\right\}\left[1-\frac{v^{2}}{c^{2}}\right] \\
\mathbf{E}_{z, \text { stat }}(t)=-\int_{0}^{L} \frac{d z}{2 \pi \varepsilon_{0} r^{2}}\left\{-\frac{\cos ^{2} \theta}{c} i\left(t^{\prime}\right)+\frac{\cos \theta}{v} i\left(t^{\prime}\right)\right\} \\
+\int_{0}^{L} \frac{d z}{2 \pi \varepsilon_{0} r^{2}}\left\{\frac{\left\{3 \sin ^{2} \theta-2\right\}}{r} \int_{t_{b}}^{t} i\left(\tau^{\prime}\right) d \tau\right\}
\end{gathered}
$$

\subsection{Continuously Moving Charge Procedure}

In the case of the transmission line model, it is a simple matter to show that the field expressions reduce to

$$
\begin{gathered}
\mathbf{E}_{z, \text { rad }}=-\frac{i\left(t^{\prime}\right) v}{2 \pi \varepsilon_{0} c^{2} d} \\
\mathbf{E}_{z, v e l}=\int_{0}^{L} \frac{d z i\left(t^{\prime}-z / v\right)\left\{1-\frac{v^{2}}{c^{2}}\right\}}{2 \pi \varepsilon_{0} r^{2}\left[1-\frac{v}{c} \cos \theta\right]^{2}}\left[\frac{\cos \theta}{v}-\frac{1}{c}\right] \\
\mathbf{E}_{z, \text { stat }}=0
\end{gathered}
$$

Note that in the case of the transmission line model, the static term and the first three terms of the radiation field reduce to zero.

\section{Discussion}

Based on the Lorentz method, the continuity equation method, the discontinuously moving charge method, and the continuously moving charge method, we have four expressions for the electric field generated by return strokes. These are the four independent methods of obtaining electromagnetic fields from the return stroke available in the literature. These expressions are given by Equations (1)-(4a-c) for the general case and Equations $(6)-(9 a-c)$, respectively, for a return stroke represented by the transmission line model. Even though the field expressions obtained by these different procedures appear different from each other, it is possible to show that they can be transformed into each other, demonstrating that the apparent non-uniqueness of the field components is due to the different ways of summing up the contributions to the total field arising from the accelerating, moving, and stationary charges.

First consider the field expression obtained using the discontinuously moving charge procedure. The expression for the total electric field is given by Equation $(8 a-c)$. In this expression, the electric fields generated by accelerating charges, uniformly moving charges, and stationary charges are given separately as Equation $(8 \mathrm{a}-\mathrm{c})$, respectively. This equation has been derived and studied in detail in $[10,12]$, and it is shown that Equation $(8 a-c)$ is analytically identical to Equation (6) derived using the Lorentz condition or the dipole procedure. Actually, this was proved to be the case for any general current distribution (i.e., for the field expressions given by Equations (1) and (3a-c)) in these publications. However, when converting Equation (8a-c) into (6) (or (3a-c) into (1)), the terms corresponding to different underlying physical processes have to be combined with each other, and the one-to-one correspondence between the electric field terms and the physical processes is lost. Moreover, observe also that the speed of propagation of the current appears only in the integration limits in Equation (1) (or (6)), as opposed to Equation (8a-c) (or (3a-c)), in which the speed appears also directly in the integrand.

Let us now consider the field expressions obtained using the continuity equation procedure. The field expression is given by Equation (7). It is possible to show that this equation is analytically equivalent to the field expression given by Equation $(9 a-c)$. This derivation is given in Appendix A.

The results discussed above show that the four field expressions given in the previous section can be reduced to two field expressions given either by Equations (6) and (7) or 
(8) and (9). In the latter set, the total field is separated into the field terms generated by accelerating charges, moving charges, and stationary charges while, in the former set, i.e., Equations (6) and (7), this connection is lost. Now, let us consider Equations (8a-c) and $(9 a-c)$. Both these equations are derived by analyzing the electromagnetic fields generated by accelerating and uniformly moving charges. Since the same charge and current distribution is assumed in both cases, one might wonder why the field equations contain different expressions for the radiation, velocity, and the static terms in the two procedures. Indeed, one would expect the same expressions for the different field components obtained using the continuously moving and discontinuously moving charge procedures. Actually, as shown in Appendix B, despite the apparent differences, Equation (8a-c) are identical to Equation $(9 \mathrm{a}-\mathrm{c})$.

The results presented above show that the field terms arising from accelerating charges, uniformly moving charges, and static charges pertinent to a given charge and current configuration can be uniquely identified. Once these field components are given, either directly or indirectly, there are many different ways to sum up these contributions and this gives rise to various techniques of electromagnetic field calculations. During this summing up procedure, the one-to-one relationship between the physical processes that give rise to the different field components is lost. Thus, different ways of summing up the contributions produce different field components giving rise to the notion of non-uniqueness of the field components arising from different techniques. In this paper, we have shown that the field components resulting from different techniques can be converted to each other, illustrating that it is the same field components but presented in a different way by combining various terms together. For example, in the dipole fields, the various field terms are combined according to the way in which the field strength is decreasing with distance. In the process, radiation, velocity, and static fields are combined with each other, and the resulting field equations do not have any resemblance to the original field terms used in the construction. A typical example for this is that of Equations (6) and (8a-c).

It is important to point out that our discussion is based on the results obtained for the transmission line model, which is a rather simple description of the spatial and temporal distribution of the return stroke current. However, any arbitrary charge and current distribution can be described as a collection of current pulses behaving exactly as in the transmission line model but displaced both spatially and in time. In order to illustrate this, consider the case in which a current pulse is moving upwards with constant speed, and it is terminated at height $z$. The same scenario can be obtained by assuming that when the current pulse reaches that point, an identical current pulse of opposite polarity will start at height $z$ and move upwards with the same speed. Thus, we will have two current pulses moving upwards with constant speed, but the total current remains zero above the height $z$. The same technique will work if the speed of the current pulse is changed at height $z$. In this case, we have to initiate two current pulses at height $z$ : one moving upwards with the reduced speed and the other moving upwards with the initial speed but with opposite polarity. This shows that any arbitrary spatial and temporal variation of the return stroke current can be described as a sum of transmission line-type currents having different speeds, polarity, and current amplitude initiated at different locations and at different times. This makes it possible to extend the results obtained here to any arbitrary current and charge distributions.

\section{Conclusions}

In the literature, there are four techniques to calculate the electromagnetic fields from lightning. These four techniques result in four expressions for the electromagnetic fields. We have shown that the field components extracted using these four techniques can be reduced to one single field expression with the total field separated into field terms arising from accelerating charges, uniformly moving charges, and stationary charges. We conclude that the non-uniqueness of the different field terms arising from different techniques is only an apparent feature. 
As long as the use of the different techniques for the field calculation is concerned, one can adopt the one that suits best the considered application (in terms of ease of application, computation time considerations, etc.), since all of them provide the same results for the total electromagnetic fields. On the other hand, if the objective is to provide insight into the underlying physical processes, the accelerating, uniformly moving, and stationary charge field components are recommended. Indeed, these components are directly related to the physical processes generating the field, and therefore, they are uniquely defined in a given reference frame.

Author Contributions: V.C. and G.C. conceived the idea and developed the mathematics and the computer software. V.C., G.C., F.R. and M.R. contributed equally to the analysis and in writing the paper. All authors have read and agreed to the published version of the manuscript.

Funding: This work was supported partly by the fund from the B. John F. and Svea Andersson donation at Uppsala University. V.C. thanks Mats Leijon for placing the research facilities of the division of electricity at V.C.'s disposal.

Conflicts of Interest: The authors declare no conflict of interest.

\section{Appendix A. Similarity of Field Expressions Given by Equations (7) and (9a-c)}

The aim of this appendix is to show analytically the equivalence between the field equations pertinent to the transmission line model derived using the continuity equation and the field equations derived using the continuously moving charge procedure. Let us start with the field equations pertinent to the continuity equation procedure. These are given by Equation (7) as

$$
E_{z}(t)=-\frac{1}{2 \pi \varepsilon_{0}} \int_{0}^{L} \frac{z}{r^{3}} \frac{i\left(t^{\prime}\right)}{v} d z-\frac{1}{2 \pi \varepsilon_{0}} \int_{0}^{L} \frac{z}{c r^{2} v} \frac{\partial i\left(t^{\prime}\right)}{\partial t} d z-\frac{1}{2 \pi \varepsilon_{0}} \int_{0}^{L} \frac{1}{c^{2} r} \frac{\partial i\left(t^{\prime}\right)}{\partial t} d z
$$

with $t^{\prime}=t-z / v-\frac{\sqrt{z^{2}+d^{2}}}{c}$.

Let us combine the last two terms of the above equation to obtain

$$
E_{z}(t)=-\frac{1}{2 \pi \varepsilon_{0}} \int_{0}^{L} \frac{z}{r^{3}} \frac{i\left(t^{\prime}\right)}{v} d z-\frac{1}{2 \pi \varepsilon_{0}} \int_{0}^{L}\left\{\frac{z}{c v\left(z^{2}+d^{2}\right)}+\frac{1}{c^{2}\left(z^{2}+d^{2}\right)^{1 / 2}}\right\} \frac{\partial i\left(t^{\prime}\right)}{\partial t} d z
$$

Now, considering $t^{\prime}=t-z / v-\frac{\sqrt{z^{2}+d^{2}}}{c}$ we find that

$$
\frac{\partial t^{\prime}}{\partial z}=\left\{-\frac{1}{v}-\frac{z}{c \sqrt{z^{2}+d^{2}}}\right\}
$$

Let us rewrite the expression for the electric field as follows

$$
\begin{aligned}
E_{z}(t)=-\frac{1}{2 \pi \varepsilon_{0}} \int_{0}^{L} & \frac{z}{r^{3}} \frac{i\left(t^{\prime}\right)}{v} d z-\frac{1}{2 \pi \varepsilon_{0}} \int_{0}^{L}\left\{\frac{z}{c v\left(z^{2}+d^{2}\right)}+\frac{1}{c^{2}\left(z^{2}+d^{2}\right)^{1 / 2}}\right\} \frac{\partial i\left(t^{\prime}\right)}{\partial t} d z \\
& -\frac{1}{2 \pi \varepsilon_{0}} \int_{0}^{L} \frac{\partial}{\partial z}\left\{i\left(t^{\prime}\right) \frac{\left[\frac{z}{c v\left(z^{2}+d^{2}\right)}+\frac{1}{c^{2}\left(z^{2}+d^{2}\right)^{1 / 2}}\right]}{\left[-\frac{1}{v}-\frac{z}{c \sqrt{z^{2}+d^{2}}}\right]}\right\} d z \\
& +\frac{1}{2 \pi \varepsilon_{0}} \int_{0}^{L} \frac{\partial}{\partial z}\left\{i\left(t^{\prime}\right) \frac{\left[\frac{z}{c v\left(z^{2}+d^{2}\right)}+\frac{1}{c^{2}\left(z^{2}+d^{2}\right)^{1 / 2}}\right]}{\left[-\frac{1}{v}-\frac{z}{c \sqrt{z^{2}+d^{2}}}\right]}\right\} d z
\end{aligned}
$$


Note that we have added and subtracted the same term from the equation. Recalling that $L=v t-r v / c$, we can solve the integration resulting in

$$
-\frac{1}{2 \pi \varepsilon_{0}} \int_{0}^{L} \frac{\partial}{\partial z}\left\{i\left(t^{\prime}\right) \frac{\left[\frac{z}{c v\left(z^{2}+d^{2}\right)}+\frac{1}{c^{2}\left(z^{2}+d^{2}\right)^{1 / 2}}\right]}{\left[-\frac{1}{v}-\frac{z}{c \sqrt{z^{2}+d^{2}}}\right]}\right\} d z=-\frac{1}{2 \pi \varepsilon_{0} c^{2}} v i(t-d / c)
$$

Thus, the expression for the electric field can be written as

$$
\begin{aligned}
& E_{z}(t)=-\frac{1}{2 \pi \varepsilon_{0}} \int_{0}^{L} \frac{z}{r^{3}} \frac{i\left(t^{\prime}\right)}{v} d z-\frac{1}{2 \pi \varepsilon_{0}} \int_{0}^{L}\left\{\frac{z}{c v\left(z^{2}+d^{2}\right)}+\frac{1}{c^{2}\left(z^{2}+d^{2}\right)^{1 / 2}}\right\} \frac{\partial i\left(t^{\prime}\right)}{\partial t} \\
& +\frac{1}{2 \pi \varepsilon_{0}} \int_{0}^{L} \frac{\partial}{\partial z}\left\{i\left(t^{\prime}\right) \frac{\left[\frac{z}{c v\left(z^{2}+d^{2}\right)}+\frac{1}{c^{2}\left(z^{2}+d^{2}\right)^{1 / 2}}\right]}{\left[-\frac{1}{v}-\frac{z}{c \sqrt{z^{2}+d^{2}}}\right]}\right\} d z-\frac{1}{2 \pi \varepsilon_{0} c^{2}} v i(t-d / c)
\end{aligned}
$$

The next step is to expand the third term into the resulting components. Let $\Lambda$ represents the third term in the above expression for the field. This can be written as

$$
\begin{aligned}
& \Lambda=\frac{1}{2 \pi \varepsilon_{0}} \int_{0}^{L}\left\{\frac{\partial i\left(t^{\prime}\right)}{\partial z} \frac{\left[\frac{z}{c v\left(z^{2}+d^{2}\right)}+\frac{1}{c^{2}\left(z^{2}+d^{2}\right)^{1 / 2}}\right]}{\left[-\frac{1}{v}-\frac{z}{c \sqrt{z^{2}+d^{2}}}\right]}\right\} d z \\
& +\frac{1}{2 \pi \varepsilon_{0}} \int_{0}^{L}\left\{i\left(t^{\prime}\right) \frac{\partial}{\partial z} \frac{\left[\frac{z}{c v\left(z^{2}+d^{2}\right)}+\frac{1}{c^{2}\left(z^{2}+d^{2}\right)^{1 / 2}}\right]}{\left[-\frac{1}{v}-\frac{z}{c \sqrt{z^{2}+d^{2}}}\right]}\right\} d z
\end{aligned}
$$

Using the relationship

$$
\frac{\partial t^{\prime}}{\partial z}=-\frac{1}{v}-\frac{z}{c \sqrt{z^{2}+d^{2}}}
$$

One can write

$$
\begin{aligned}
& \Lambda=\frac{1}{2 \pi \varepsilon_{0}} \int_{0}^{L}\left\{\frac{\partial i\left(t^{\prime}\right)}{\partial t^{\prime}}\left[\frac{z}{c v\left(z^{2}+d^{2}\right)}+\frac{1}{c^{2}\left(z^{2}+d^{2}\right)^{1 / 2}}\right]\right\} d z \\
& +\frac{1}{2 \pi \varepsilon_{0}} \int_{0}^{L}\left\{i\left(t^{\prime}\right) \frac{\partial}{\partial z} \frac{\left[\frac{z}{c v\left(z^{2}+d^{2}\right)}+\frac{1}{c^{2}\left(z^{2}+d^{2}\right)^{1 / 2}}\right]}{\left[-\frac{1}{v}-\frac{z}{c \sqrt{z^{2}+d^{2}}}\right]}\right\} d z
\end{aligned}
$$

Substituting this into the expression for the field, we obtain

$$
\begin{gathered}
E_{z}(t)=-\frac{1}{2 \pi \varepsilon_{0}} \int_{0}^{L} \frac{z}{r^{3}} \frac{i\left(t^{\prime}\right)}{v} d z+\frac{1}{2 \pi \varepsilon_{0}} \int_{0}^{L}\left\{i\left(t^{\prime}\right) \frac{\partial}{\partial z} \frac{\left[\frac{z}{c v\left(z^{2}+d^{2}\right)}+\frac{1}{c^{2}\left(z^{2}+d^{2}\right)^{1 / 2}}\right]}{\left[-\frac{1}{v}-\frac{z}{\left.c \sqrt{z^{2}+d^{2}}\right]}\right\} d z}\right. \\
-\frac{1}{2 \pi \varepsilon_{0} c^{2}} v i(t-d / c)
\end{gathered}
$$

In order to limit the number of expressions to be written, let us write the above equation as

$$
E_{z}(t)=-\frac{1}{2 \pi \varepsilon_{0}} \int_{0}^{L} F_{1} d z+\frac{1}{2 \pi \varepsilon_{0}} \int_{0}^{L} F_{2} d z-\frac{1}{2 \pi \varepsilon_{0} c^{2}} v i(t-d / c)
$$


In the above equation, $F_{1}=i\left(t^{\prime}\right) \frac{\cos \theta}{v r^{2}}$ and the function $F_{2}$ is given by

$$
\begin{gathered}
F_{2}=-i\left(t^{\prime}\right) \frac{\cos \theta}{c v r} \frac{v^{2}}{\left(1-\frac{v}{c} \cos \theta\right)^{2}} \frac{1}{c r}\left(1-\cos ^{2} \theta\right)+\frac{i\left(t^{\prime}\right)}{c^{2} r} \frac{v^{2}}{\left(1-\frac{v}{c} \cos \theta\right)^{2}} \frac{1}{c r}\left(1-\cos ^{2} \theta\right) \\
-\frac{i\left(t^{\prime}\right)}{c v r^{2}}\left(1-2 \cos ^{2} \theta\right) \frac{v}{\left(1-\frac{v}{c} \cos \theta\right)}-\frac{i\left(t^{\prime}\right) \cos \theta}{v r^{2}} \frac{v}{\left(1-\frac{v}{c} \cos \theta\right)}
\end{gathered}
$$

Now, multiplying up and down of the second and the fourth term given above by $(1-v \cos \theta / c)$, multiplying $F_{1}$ up and down by $(1-v \cos \theta / c)^{2}$ and combining the terms, we obtain

$$
-F_{1}+F_{2}=i\left(t^{\prime}\right) \frac{1}{v}\left(1-\frac{v^{2}}{c^{2}}\right) \frac{1}{r^{2}\left(1-\frac{v}{c} \cos \theta\right)^{2}}\left(\cos \theta-\frac{v}{c}\right)
$$

So, the expression for the electric field reduces to

$$
E_{z}(t)=\frac{1}{2 \pi \varepsilon_{0} v}\left(1-\frac{v^{2}}{c^{2}}\right) \int_{0}^{L} \frac{i(t-z / v-r / c)\left(\cos \theta-\frac{v}{c}\right)}{r^{2}\left(1-\frac{v}{c} \cos \theta\right)^{2}} d z-\frac{1}{2 \pi \varepsilon_{0} c^{2}} v i(t-d / c)
$$

This expression for the field is identical to the expression derived using the continuously moving charge method.

\section{Appendix B. Similarity of the Field Expressions Given by Equations (8a-c) and (9a-c)}

In order to prove that the field terms in Equations $(8 \mathrm{a}-\mathrm{c})$ and $(9 \mathrm{a}-\mathrm{c})$ are identical to each other, it is necessary to go back to the original derivation of Equation ( $8 \mathrm{a}-\mathrm{c})$. First of all, observe that the velocity terms are the same in both equations, and we only have to prove the identity of the radiation and static fields. Of course, there may be a straightforward way to show that the field terms are identical, but we were unable to find that shortcut. Equation ( $8 \mathrm{a}-\mathrm{c}$ ) was derived by evaluating the electric field produced by a channel element using the charge acceleration equations and then summing the contribution from all the channel elements. Let us now follow the steps necessary in this derivation.

\section{Appendix B.1. Electromagnetic Fields Generated by a Channel Element}

Divide the channel into a large number of small elements of length $d z$. Consider the channel element located at height $z$ along the channel. An expanded view of this channel element together with the geometry necessary for the mathematical derivation is depicted in Figure A1. Then, the first step is to estimate the electromagnetic fields generated by the said channel element. We consider the transmission line model of the return stroke and, hence, we represent the current flowing along the channel element by $i(t-z / v)$. In writing down the equations corresponding to the field components, we treat the current flow along the element in such a way that it is initiated at the bottom of the channel element and is absorbed at the upper end. Thus, the current that appears at the bottom of the channel element at any time $t$ will appear at the top of the channel element after a time delay given by the ratio of the length and the speed, $d z / v$.

The electromagnetic fields generated by the channel element can be divided into different components as follows: (a) the electric and magnetic radiation fields generated at the initiation and termination of the current at the end points of the channel element due to charge acceleration and deceleration, respectively; (b) the electric and magnetic velocity fields generated by the movement of charges along the channel element; (c) the static field generated by the accumulation of charges at the two ends of the channel element. Let us consider these different field components separately. In writing down these field components, we will depend heavily on the results published previously by Cooray and Cooray [10,12]. 


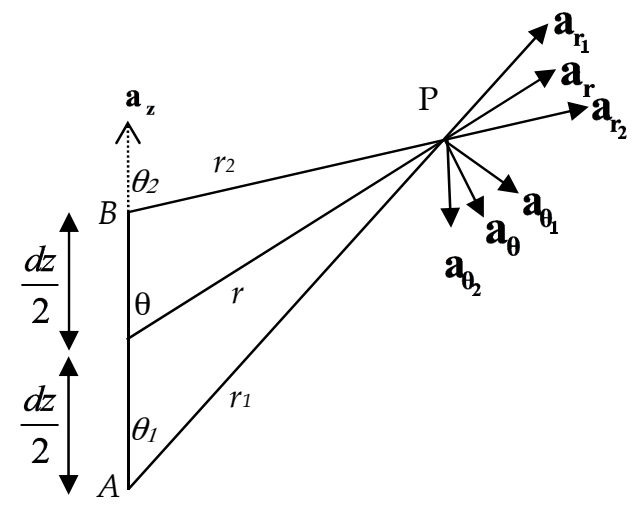

Figure A1. Geometry, angles and unit vectors pertinent to the evaluation of electromagnetic fields generated by a channel element. The unit vector in the direction of the positive $z$-axis is denoted by $\mathbf{a}_{\mathbf{z}}$. The unit vectors in the radial directions $r, r_{1}$ and $r_{2}$ are denoted by $\mathbf{a}_{r}, \mathbf{a}_{r_{1}}$ and $\mathbf{a}_{r_{2}}$ respectively. The unit vectors $\mathbf{a}_{\theta}, \mathbf{a}_{\theta_{1}}$ and $\mathbf{a}_{\theta_{2}}$ are defined as $\mathbf{a}_{r} \times\left(\mathbf{a}_{r} \times \mathbf{a}_{z}\right), \mathbf{a}_{r_{1}} \times\left(\mathbf{a}_{r_{1}} \times \mathbf{a}_{z}\right)$ and $\mathbf{a}_{r_{2}} \times\left(\mathbf{a}_{r_{2}} \times \mathbf{a}_{z}\right)$, respectively. Note that the point $P$ can be located anywhere in space.

Appendix B.1.1. Radiation Field Generated by the Charge Acceleration and Deceleration at the Ends of the Channel Element

The electric radiation field generated by the initiation of the current at the bottom of the channel element and by the termination of that current at the top of the channel element is given by

$$
d \mathbf{e}_{r a d}=\frac{v}{4 \pi \varepsilon_{0} c^{2}}\left\{\frac{i\left(t-z / v-r_{1} / c\right) \sin \theta_{1}}{r_{1}\left[1-\frac{v \cos \theta_{1}}{c}\right]} \mathbf{a}_{\theta_{1}}-\frac{i\left(t-z / v-d z / v-r_{2} / c\right) \sin \theta_{2}}{r_{2}\left[1-\frac{v \cos \theta_{2}}{c}\right]} \mathbf{a}_{\theta_{2}}\right\}
$$

Appendix B.1.2. Electrostatic Field Generated by the Accumulation of Charge at A and B

As the positive current leaves point $A$, negative charge accumulates at $A$, and when the current is terminated at $B$, positive charge is accumulated there. The static Coulomb field produced by these stationary charges is given by

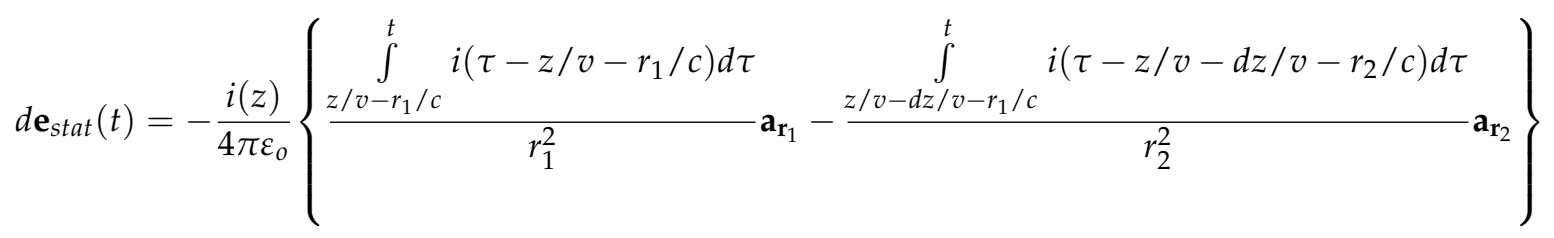

\section{Appendix B.2. Electromagnetic Fields Generated by the Lightning Channel}

The radiation and static terms in Equation $(8 \mathrm{a}-\mathrm{c})$ follow directly from the above two equations A15 and A16 once the equations are reduced for the condition that $d z$ is infinitesimal and summing up the contribution from all the channel elements by performing the integration along the channel. However, let us keep the above equations in the current form and replace the integration along the channel by a summation. Let us consider the radiation field. When we take the summation starting from the first element located at the bottom of the channel, one can see directly that the radiation coming from the top of the first element will be cancelled off with the radiation coming from the bottom of the second element, the radiation coming from the top of the second element will be cancelled off with the radiation coming from the bottom of the third element, etc. As a result, at any point in space, only the radiation term coming from the bottom of the first element will survive 
during the summation. Thus, the radiation field at the surface of a perfectly conducting ground is given by

$$
\mathbf{e}_{\text {rad }}=-\frac{v}{2 \pi \varepsilon_{0} c^{2}} \frac{i(t-z / v-d / c)}{d} \mathbf{a}_{\mathbf{z}} .
$$

Observe that in the above case, $r_{1} \rightarrow D, \sin \theta \rightarrow 1$ and $\mathbf{a}_{\theta} \rightarrow-\mathbf{a}_{z}$. This is identical to the radiation field in Equation $(9 \mathrm{a}-\mathrm{c})$. Now, let us consider the static term. As in the radiation field, when you take the summation, only the term corresponding to the bottom of the first element will survive. However, when we take into account the fact that the lightning channel is located above a perfectly conducting ground, this static term will cancel off with the corresponding term associated with the image of the element in the perfectly conducting ground plane. Thus, the total static field will become equal to zero. That is,

$$
\mathbf{e}_{\text {stat }}=0 \text {. }
$$

This analysis shows that all the terms of Equation $(8 \mathrm{a}-\mathrm{c})$ are identical to the corresponding terms in Equation $(9 \mathrm{a}-\mathrm{c})$ and that these two equations are identical to each other. Just to illustrate this further, we have calculated the electric field at $100 \mathrm{~m}$ distance from a lightning channel using Equations $(8 a-c)$ and $(9 a-c)$. The different components and the total field obtained from Equations $(8 a-c)$ and $(9 a-c)$ are depicted in Figure A2. Note that as illustrated above, the three field terms are identical in both formulations.

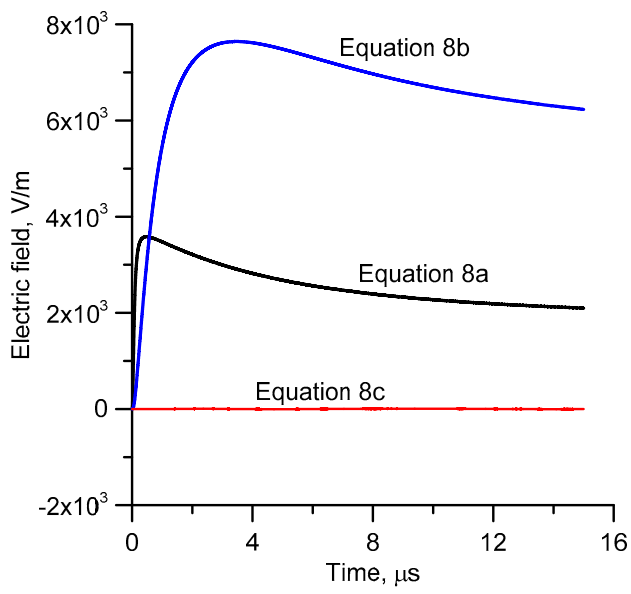

(a)

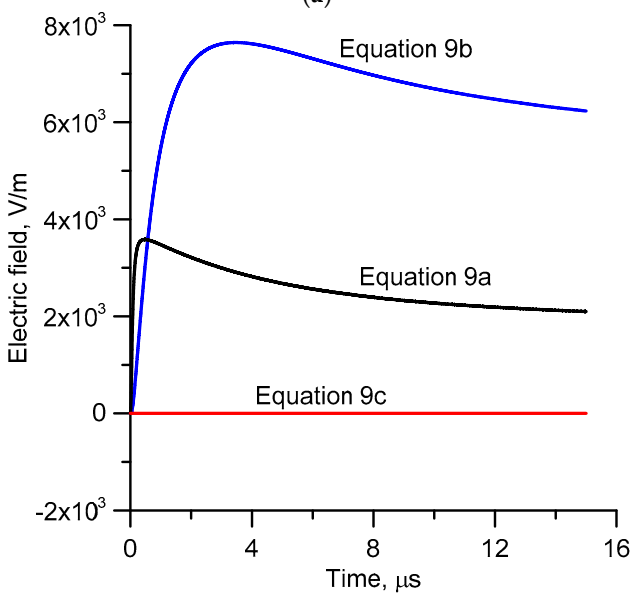

(b)

Figure A2. Plot of the field components associated with (a) Equation (8a-c) and (b) Equation (9a-c). The electric field is calculated at $100 \mathrm{~m}$ from the strike point of a lightning return stroke simulated by the transmission line model. The current at the channel base is represented by the analytical expression given by Nucci et al. [17] to represent subsequent return strokes. The return stroke speed used in the calculation is $1.5 \times 10^{8} \mathrm{~m} / \mathrm{s}$. 


\section{References}

1. Jackson, J.D. Classical Electrodynamics, 3rd ed.; Wiley: Hoboken, NJ, USA, 1998.

2. Lin, Y.T.; Uman, M.A.; Standler, R.B. Lightning return stroke models. J. Geophys. Res. Space Phys. 1980, 85, 1571-1583. [CrossRef]

3. Rubinstein, M.; Uman, M. Methods for calculating the electromagnetic fields from a known source distribution: Application to lightning. IEEE Trans. Electromagn. Compat. 1989, 31, 183-189. [CrossRef]

4. Safaeinili, A.; Mina, M. On the analytical equivalence of electromagnetic fields solutions from a known source distribution. IEEE Trans. Electromagn. Compat. 1991, 33, 69-71. [CrossRef]

5. Thottappillil, R.; Rakov, V.; Uman, M.A. Distribution of charge along the lightning channel: Relation to remote electric and magnetic fields and to return-stroke models. J. Geophys. Res. Space Phys. 1997, 102, 6987-7006. [CrossRef]

6. Thomson, E.M. Exact expressions for electric and magnetic fields from a propagating lightning channel with arbitrary orientation. J. Geophys. Res. Space Phys. 1999, 104, 22293-22300. [CrossRef]

7. Shao, X. Generalization of the lightning electromagnetic equations of Uman, McLain, and Krider based on Jefimenko equations. J. Geophys. Res. Atmos. 2016, 121, 3363-3371. [CrossRef]

8. Thottappillil, R.; Rakov, V.A. On different approaches to calculating lightning electric fields. J. Geophys. Res. Space Phys. 2001, 106, 14191-14205. [CrossRef]

9. Cooray, V.; Cooray, G. The Electromagnetic Fields of an Accelerating Charge: Applications in Lightning Return-Stroke Models. IEEE Trans. Electromagn. Compat. 2010, 52, 944-955. [CrossRef]

10. Cooray, G.; Cooray, V. Electromagnetic Fields of a Short Electric Dipole in Free Space-Revisited. Prog. Electromagn. Res. 2012, 131, 357-373. [CrossRef]

11. Cooray, V.; Cooray, G. Electromagnetic fields of accelerating charges: Applications in lightning protection. Electr. Power Syst. Res. 2017, 145, 234-247. [CrossRef]

12. Cooray, V.; Cooray, A.G. A Novel Interpretation of the Electromagnetic Fields of Lightning Return Strokes. Atmosphere 2019, 10, 22. [CrossRef]

13. Cooray, V.; Cooray, G.; Rubinstein, M.; Rachidi, F. Generalized Electric Field Equations of a Time-Varying Current Distribution Based on the Electromagnetic Fields of Moving and Accelerating Charges. Atmosphere 2019, 10, 367. [CrossRef]

14. Rubinstein, M.; Uman, M.A. On the radiation field turn-on term associated with traveling current discontinuities in lightning. J. Geophys. Res. Space Phys. 1990, 95, 3711-3713. [CrossRef]

15. Uman, M.A.; McLain, D.K. Magnetic field of lightning return stroke. J. Geophys. Res. Space Phys. 1969, 74, 6899-6910. [CrossRef]

16. Thottappillil, R. Computation of electromagnetic fields from lightning discharge. In The Lightning Flash; Cooray, V., Ed.; IEE Press: London, UK, 2003.

17. Nucci, C.A.; Diendorfer, G.; Uman, M.A.; Rachidi, F.; Ianoz, M.; Mazzetti, C. Lightning return stroke current models with specified channel-base current: A review and comparison. J. Geophys. Res. Space Phys. 1990, 95, 20395-20408. [CrossRef] 